\title{
SSRI-Induced Activation Syndrome in Children and Adolescents-What Is Next?
}

Maya Amitai, $M D^{1,2, *}$

Alon Chen, $P h D^{3,4}$

Abraham Weizman 2,5

Alan Apter, M.D. ${ }^{1,2}$

\author{
Address \\ ${ }^{*}, 1$ Department of Psychological Medicine, Schneider Children's Medical Center of \\ Israel, 14 Kaplan Street P0 Box 559, Petah Tikva, 49202, Israel \\ Email: maya47@zahav.net.il \\ ${ }^{2}$ Sackler Faculty of Medicine, Tel Aviv University, Tel Aviv, Israel \\ Email: asapter@gmail.com \\ ${ }^{3}$ The Ruhman Family Laboratory for Research on the Neurobiology of Stress, \\ Department of Neurobiology, Weizmann Institute of Science, 76100, Rehovot, \\ Israel \\ Email: Alon.Chen@weizmann.ac.il \\ ${ }^{4}$ Department of Stress Neurobiology and Neurogenetics, Max-Planck Institute of \\ Psychiatry, 80804, Munich, Germany \\ Email: Alon.Chen@weizmann.ac.il \\ ${ }^{5}$ Research Unit, Geha Mental Health Center, Petah Tikva, Israel \\ Email: AWeizman@clalit.org.il
}

Published online: 30 January 2015

(C) Springer International Publishing AG 2015

This article is part of the Topical Collection on Child and Adolescent Psychiatry

Keywords SSRI - Children · Adolescents - Adverse events - Activation syndrome

\section{Opinion statement}

Antidepressants are being increasingly prescribed for children and adolescents for the treatment of depression, anxiety, and other mental disorders. The selective serotonin reuptake inhibitors (SSRIs) are the major contributors to this trend. Children are especially prone to psychiatric adverse events of SSRIs relative to adults. Recently, attention has been addressed to a specific constellation of emotional and behavioral symptoms associated with SSRI treatment, termed activation syndrome. However, the prevalence, characteristics, and implications of this syndrome have not been systematically examined in the pediatric population. Furthermore, the relationship between SSRI-induced activation and bipolar disorder or other psychiatric adverse events, particularly suicidality and manic conversion, remains unclear. The aim of this review was to report on the current scientific 
literature on SSRI-induced activation syndrome in young patients and to suggest future directions for research. This information will help to clarify the underlying mechanism of this phenomenon and its prognostic significance and aid clinicians in identifying patients at risk and developing effective management and preventive measures.

\section{Introduction}

Antidepressant medications are used since 1950s for the treatment of depression, anxiety, obsessive-compulsive disorder, and other mental disorders [1]. Though originally intended for adults, they are currently widely prescribed to children and adolescents [2]. The selective serotonin reuptake inhibitors (SSRIs), including fluoxetine, paroxetine, sertraline, fluvoxamine, citalopram, and escitalopram, are the major contributors to this upward trend. SSRIs are at present the most commonly used psychotropics in the pediatric population [3].

The general efficacy and safety of SSRIs have been supported by large controlled single-site and multisite studies [4]. However, given the known developmental differences in pharmacodynamics and pharmacokinetics, the responses to SSRIs in children and adolescents are different than in adults. For example, tricyclic antidepressants are effective for adult depression but not for adolescent depression [5]. Furthermore, age is known to be a significant factor in the emergence of some adverse effects of antidepressants [6, 7].

Particularly worrisome is the association of SSRIs with psychiatric adverse events. These have been categorized into several spectrums: manic symptoms (mania, hypomania, elevated mood), depressive symptoms (aggravation of depression, crying, irritability, anger, hypersensitivity), agitative symptoms (agitation, akathisia, restlessness, nervousness hyperactivity), anxiety and panic symptoms, apathy, tremor, and "feeling spacy" $[8,9]$. In recent years, attention has been addressed to a cluster of symptoms of excessive emotional arousal or behavioral activation, termed activation syndrome, which may appear during the course of SSRI treatment [10]. However, data remain sparse, particularly in children and adolescents, as most drug studies in this age group have focused on efficacy and were not designed to systematically investigate such side effects [8]. The precise definition of the syndrome, the impact of time of administration, dosage, and patient characteristics on its occurrence, and the underlying mechanism(s) are still unclear. This has raised concerns in light of the considerable impact of the syndrome on both patient and family, in addition to its potential association with bipolar disorder $[11 \bullet, 12]$ and other psychiatric adverse effects of SSRIs such as mania and suicidality [13]. Furthermore, activation syndrome has been cited a major reason for withdrawal from clinical trials in children $(12.1 \%)$ and adolescents $(7.6 \%)[7,14,15]$.

This review summarizes the contemporary medical literature on the association of SSRI treatment with the emergence of activation syndrome in children and adolescents and to suggest future directions for understanding this serious phenomenon.

\section{Characterization of SSRI-induced activation syndrome}

Symptoms and signs

The psychiatric signs and symptoms include irritability, agitation, somatic manifestations of anxiety, panic attacks, restlessness, hostility, aggression, insomnia, disinhibition, emotional liability, impulsivity, social withdrawal, akathisia (psychomotor restlessness), odd behavior, hypomania/mania, and paranoia or other psychotic symptoms $[1,16,17]$. Activation syndrome may be defined, broadly or narrowly, depending on how many constructs are included. Even when akathisia and mania are excluded, the collection of signs and symptoms described under the rubric of activation syndrome may not represent a single diathesis $[11 \bullet]$. Some of them may not be caused by 
the drug per se but rather manifestations of deteriorating or evolution of the underlying psychiatric condition, indicating resistance to the drug.

Time of onset after SSRI administration

Although it has been reported that activation can occur at any time during the course of treatment, there seems to be a tendency for it to appear during the first 2 or 3 weeks after drug initiation $[1,18]$. These findings have been tied to studies of suicidal risk in relation to SSRI treatment, although this remains controversial $[2,16]$. This issue is discussed in detail in the following sections.

Relationship to drug dose

Some studies found no relationship between the SSRI dose and the appearance of activation syndrome [19], whereas others reported that children treated with a lower dose of SSRI are more prone to behavioral activation [20]. However, most studies suggest that higher doses are associated with higher rates of activation $[7,14]$. Conversely, it is possible that reducing the dose may have a beneficial effect [14].

Biological susceptibility

Activation is more common in children and adolescents than adults and up to twice as common in children than adolescents [7]. A recent naturalistic study reported that patients who suffered from such adverse events were significantly younger than those who did not $(p=0.02)$, and every 1 -year increase in age was associated with a $27 \%$ decrease in the probability of a severe event [21•]. A biological susceptibility to activation syndrome [20] is supported by preclinical studies wherein prepubertal rodents administered SSRIs displayed increased density of the serotonin transporters in the frontal cortex that persisted into maturity [22]. In addition, their response to serotonergic and noradrenergic probes was dramatically different when they were at their prepubertal age [23].

\section{Prevalence of activation syndrome}

The exact prevalence of SSRI-induced activation in pediatric age group is unclear. A retrospective chart review of 82 children and adolescents with depression or obsessive-compulsive disorder documented psychiatric adverse events, mainly mood disturbances, in $22 \%$ [24]. In $44 \%$, reexposure to an SSRI caused another psychiatric adverse event [25]. In a prospective study in 24 children and adolescents with obsessivecompulsive disorder treated with fluoxetine, $50 \%$ had activation symptoms [10]. A larger, multisite, placebo-controlled trial of patients aged 6-7 years with anxiety disorders reported increased behavioral symptoms in $27 \%$ of the fluvoxamine-treated group and $12 \%$ of the placebo group; however, this difference was not statistically significant 
[26]. However, since the analysis was limited to motor activity, the occurrence of activation syndrome may have been underestimated.

In a study based on the double-blind placebo-controlled Research Unit on Pediatric Psychopharmacology Anxiety Study, 10 of 22 youths $(45 \%)$ treated with fluvoxamine for anxiety experienced symptoms of activation syndrome compared to only 1 of 23 youths $(4 \%)$ in the placebo group. Mean fluvoxamine blood level at week 8 was significantly higher in the subjects who had adverse events than in those who did not [20]. This is the only research to our knowledge that compared SSRI blood levels between treated youths with and without SSRI-induced activation syndrome.

According to a review study of a pediatric, double-blind, a placebocontrolled trial of SSRIs, the mean prevalence rate of activation syndrome was $10.7 \%$ in children and $2.1 \%$ in adolescents [7]. A larger, more recent meta-analysis of adverse events associated with antidepressants in youths [11•] found that in the subjects with depressive disorders, mean rates of apparent mood shifting were 20.5 times higher in the antidepressant than the placebo arm, and in the subjects with anxiety disorders, rates of apparent arousal-activation responses were 3.13 times higher in the antidepressant than the placebo arm. Interestingly, on analysis by primary diagnosis, the risk was at least as high in anxiety as depressive disorders. Analysis by symptom showed that for mania-hypomania specifically, the drug-associated risk was $8.19 \%$ compared to $0.17 \%$ for placebo, a 45 -fold difference. Overall, the risk of arousal activation, notwithstanding the main diagnosis or outcome, was $12.9 \%$ for antidepressant treatment and $3.69 \%$ for placebo-a highly significant 3.49-fold difference (drug/placebo risk ratio, 1.66). The estimated number-needed-to-harm (NNH) to obtain one case of unwarranted arousal with an antidepressant versus placebo was 37 for depressed and 32 for anxious patients.

Prevalence rates of activation syndrome are also higher in certain subgroups of patients. Open-label and case series report high rates (more than $20 \%$ ) in children with mental retardation, autism, Tourette's syndrome, panic disorder, and pervasive developmental disorder [7].

A more accurate definition of SSRI-induced activation syndrome is needed to narrow the wide variation in reported prevalence rates in the literature.

\section{Akathisia and SSRI-induced activation syndrome}

There is no agreement regarding the distinction between activation syndrome from akathisia (psychomotor restlessness). Although antipsychotics are typically responsible for the occurrence of akathisia, SSRIs have been implicated as well $[27,28]$. Akathisia can be separated into subjective (e.g., inner restlessness and urge to move) and objective (e.g., knee nodding while sitting) components [1, 29]. There is a possible connection between akathisia and suicidality $[27,28]$. Akathisia is often included as a component of activation syndrome. However, the two phenomena are not the same since the former, namely antipsychoticinduced akathisia, suggests a known neuroreceptor mechanism (e.g., 
dopamine-receptor blockade), while the dopamine role is unknown in SSRI-induced activation syndrome.

\section{Mania and SSRI-induced activation syndrome}

It is common knowledge that antidepressant medications, particularly SSRIs, can precipitate manic spectrum symptoms in susceptible individuals [6]. Mild hypomanic symptoms are much more common than manic switching [8], defined as a single transition from depression to mania temporally related to antidepressant use. According to data derived from a national administrative database, rates of antidepressant-induced manic switching in naturalistic and controlled studies range from 7 to $10 \%$; they may reach as high as $67 \%$ in patients with bipolar disorder $[6,8]$. Within the pediatric population, the reported rate is $5.4 \%$, with peripubertal children at highest risk $(\mathrm{NNH}=10$ in the 10- to 14-year-old group compared to 23 in the 15- to 29-year-old group) [6]. Randomized controlled trials in children and adolescents showed a $\leq 2 \%$ risk, at least in the short-to-medium treatment term [4].

Data relating to SSRI-induced activation syndrome in this context were provided by a recent prospective study of the safety and tolerability of SSRIs in children and adolescents (age 9-20 years) with depressive or anxiety disorders and a family history (at least one parent) of bipolar disorder [21 $\bullet$ [class II]. An adverse drug reaction, namely, irritability, aggression, impulsivity, or hyperactivity, that led to trial discontinuation occurred in $57 \%$ of the study group; the rate was significantly higher in the younger patients $(p=0.02)$. These data suggest that behavioral activation might represent subsyndromal manic symptoms or unrecognized bipolar disorder, especially in young persons who have not been diagnosed. However, there is still a possibility that antidepressants cause de novo induction of bipolar disorder $[11 \bullet, 12]$. Some researchers stress that SSRI-induced manic symptoms and the activation syndrome should be distinguished since manic spectrum symptoms involve a change in mood and behavior with coexisting symptoms of grandiosity and euphoria which do not exist in the SSRI-induced activation syndrome [25].

Clearly more knowledge regarding the pathophysiology of the activation syndrome will clarify the connection between these two SSRI-induced phenomena.

\section{Suicidality and SSRI-induced activation syndrome}

Controlled clinical trials have reported an association between antidepressant use and an increased rate of suicidality in youth [16, 30, 31•]. In 2004, drug regulatory agencies in the USA (Food and Drug Administration), Canada (Health Canada), and the UK (Medicines and Healthcare Regulatory Agency) published a "black box" warning that in children and adolescents, treatment with antidepressants may pose a risk of suicidality, violence, aggression, mania, and other abnormal behavioral changes [4]. This was followed by a decrease in prescription rates [32], as it raised many questions regarding the appropriate circumstances for prescribing antidepressants, with a need to carefully weigh the benefits and risks in each individual case. 
The mechanism whereby antidepressants increase suicidal ideation and suicidal behavior is unknown. Researchers speculate that activation syndrome may be related to suicidality, but the evidence is scant [13].

In line with the suggestion that antidepressants produce a "behavioral toxicity" in susceptible individuals [33], suicidality in this setting may be a consequence of the drug-induced behavioral activation, including anxiety, irritability, agitation, and insomnia [11•]. The suicidality-activation link is further supported by the analysis of suicidality in the FDA review of placebocontrolled, clinical trials in youth, which showed that those who experienced the activation syndrome were two to three times more prone to suffer from suicidality [7]. This association is further supported by the findings that suicidal risk is higher in the first week of antidepressant treatment, the same as the risk of activation syndrome [2]. However, the large (439 participants) 36-week randomized controlled Treatment for Adolescents with Depression Study (TADS) [34] yielded opposing results. One aim of the TADS was to identify possible indicators of antidepressant-induced behavioral activation syndrome preceding suicidal events. There was no evidence of an increase in irritability, mood instability, insomnia, and akathisia within 2 weeks prior to the suicidal event, suggesting that SSRI-induced behavioral activation is not a trigger of suicidal behavior.

Taking another approach, some researchers suggested that juveniles who are not forewarned of the possibility of activation syndrome under treatment with an antidepressant may misinterpret these effects as deterioration in their underlying condition. They may conclude that the treatment is futile, plunging them into despair and hopelessness, leading to suicidality [11 $\bullet$.

It is important to mention that activation syndrome should be distinguished from energizing phenomena, first described by Mayer-Gross in 1960 [11•]. This phenomenon is described when a depressed patient (particularly one with psychomotor retardation) harbors suicidal thoughts but have no energy to perform the self-injurious act. During the early phase of antidepressant treatment, when patient energy is boosted but the depressive mood has not yet lifted, there is a higher risk that the patient will perform the suicidal act. In this context, the antidepressant does not cause suicidality per se but rather restores the lacking energy. Thus, conceptually, wherein activation syndrome implies (at least temporary) a behavioral adverse event, energizing phenomena imply that the medication is successful however mood has yet to respond. Therefore, though the distinction between the entities is operationally difficult, a different clinical management approach is needed for each.

At present, there is a great need to appropriately dose SSRIs in order to lower the risk of SSRI-induced activation syndrome [19, 35]. There is a crucial need to clarify the possible association between activation syndrome and SSRI-induced suicidality so that preventive measures may be employed and improved.

\section{SSRI pharmacokinetics and pharmacogenetics and activation syndrome}

SSRIs have a distinct profile for cytochrome inhibition. Fluoxetine and paroxetine are potent CYP2D6 inhibitors; therefore, there is a possibility that reduced 
clearance of these drugs in slow metabolizers of the cytochrome P450 2D6 isoenzyme leading to higher plasma (and brain) levels with the same dose [36]. A study by our group of the association between polymorphism in the serotonin transporter gene promoter (5-HTTLPR) and citalopram effectiveness and adverse events in children and adolescents with major depressive and/or anxiety disorders yielded less agitation in those with the 5-HTTLPR ss genotype than in those with the sl/ll genotype [37].

Together, these findings suggest that individual variations in either drug pharmacokinetics (e.g., slow hepatic biotransformation) or pharmacodynamics (e.g., serotonin transporter polymorphisms) cause individual reactions to the same drugs. The pharmacokinetic factors can be approached by lowering the dose or selecting a different agent. More information is required to examine the role of SSRI pharmacogenomics in the pediatric age group. Pretreatment genetic testing before starting therapy would then make it possible for clinicians to better predict which patients are more susceptible to side effects.

\section{Pathophysiology of activation syndrome}

Several potential mechanisms have been proposed regarding the pathophysiology of activation syndrome $[5,20]$. A movement disorder, parallel to akathisia, may lead to heightened aggression, impulsivity, and suicidality, especially if mood has not yet improved [38]. Switch to a variant of a manic or mixed state is also a possible explanation [25]. Additionally, the excessive arousal activation in response to SSRIs may be a manifestation of bipolar disorder, especially in juveniles at risk with no history of mania or hypomania [28]. Indeed, it has been suggested that these events may serve as a clinical basis for recognizing bipolar disorder in depressed patients $[6,11 \bullet, 12,39,40]$, which is particularly pertinent in juveniles in whom bipolar disorder is not easily diagnosed [11 $\bullet$ 41-43]. Finally, the excessive mood elevation may be a direct pharmacologic effect of SSRIs [36], with a high risk in children and adolescents [20].

Activation syndrome may be related to self-injury via diminished serotonergic activity which could compromise the ability of patients to self-regulate their behavior $[9,19]$. The activation-related disinhibition may also lead to impulse control problems [27]. Preclinical trials do not report a decrease in serotonin activity during the first days or weeks of SSRI treatment [44]; however, it is possible that other changes in regional brain 5-HT2 or 5-HT3 receptor sensitivity might be involved [17].

\section{Clinical/Biological redictors of SSRI-induced activation}

A complex interaction between genetic predisposition and environmental factors is suggested to be involved in response to drugs. In recent years, there has been an increased interest in elucidating the role of epigenetic processes in mental disorders and response to therapy. One of the mechanisms suggested to be involved in epigenetic processes is the regulation by non-coding RNAs such as microRNAs (miRNAs). Studies on the role of miRNAs in psychopathologies involve data collected from human patients, animal models for mental disorders, and cellular systems and have used a variety of approaches. Blood levels of 


\section{Conclusion}

miRNAs in patients have been tested as potential biomarkers to predict treatment response. An example is a study reporting that blood miR-1202 levels could distinguish between SSRI responders and non-responders; specifically, miR-1202 levels are initially lower and are upregulated upon drug administration in responders but not in non-responders [45]. This suggests that circulatory miR-1202 levels could be potentially used to predict possible side effects (including SSRI-induced activation syndrome). Thus, blood levels of miRNAs may serve as potential biomarkers in predicting SSRI-induced activation syndrome.

Our group is currently studying SSRI-induced adverse events in a population of children and adolescents, with close monitoring for possible side effects. The aim is to identify biomarkers predicting adverse events, including SSRI-induced activation syndrome. So far, we have recruited 70 patients with depression and/or anxiety disorders treated with SSRIs for 1 year. Nine patients displayed SSRI-induced activation syndrome that led to drug cessation. One 15-year-old boy made a suicide attempt which was preceded by irritability and heightened mood, and one 14-year-old girl had an episode of manic switching. These observations should have significant implications for the future development of novel diagnostic tests so that safer treatment can be offered to these young patients.

There is a clear and considerable risk of excessive arousal or activation, and even hypomania or mania, in children and adolescents treated with SSRIs. The rates of these symptoms, even those diagnosed as mania or hypomania, are much higher than in adults with anxiety and depression. To illuminate the mechanism and prognostic significance of excessive arousal-activation associated with SSRI treatment in children and adolescents, more studies, including long term follow-up of affected patients, are needed. Better understanding of activation syndrome may reveal the neurobiological mediators of this adverse effect and hopefully will lead to identification of effective strategies for prevention. Further empirical research will aid clinicians in prospectively identifying individuals at increased risk.

At present, we recommend close clinical monitoring during SSRI initiation or dose changes, which should reduce the risk of activation syndrome and more importantly recognizing it on time. Particular caution is recommended in children and adolescents at risk of having undiagnosed bipolar disorder. Attention should be paid to the emergence of bipolar disorder and future responses to SSRIs in patients with pathological mood elevation or behavioral activation.

\section{Compliance with Ethics Guidelines}

\section{Conflict of Interest}

Maya Amitai declares no conflict of interest. Alon Chen declares no conflict of interest. Abraham Weizman declares no conflict of interest. Alan Apter declares no conflict of interest. 
Human and Animal Rights and Informed Consent

This article does not contain any studies with animal subjects performed by any of the authors.

\section{References and Recommended Reading}

Papers of particular interest, published recently, have been highlighted as:

- $\quad$ Of importance

1. Murray ML, de Vries CS, Wong IC. A drug utilisation study of antidepressants in children and adolescents using the general practice research database. Arch Dis Child. 2004;89:1098-102.

2. Simon GE, Savarino J, Operskalski B, Wang PS. Suicide risk during antidepressant treatment. Am J Psychiatry. 2006;163:41-7.

3. Zito JM, Tobi H, De Jong-van den Berg LT, Fegert JM, Safer DJ, Janhsen K. Antidepressant prevalence for youths: a multi-national comparison.

Pharmacoepidemiol Drug Saf. 2006;15:793-8.

4. Cheung AH, Emslie GJ, Mayes TL. Review of the efficacy and safety of antidepressants in youth depression. J Child Psychol Psychiatry. 2005;46:735-54.

5. Hazell P,O'Connell D, Heathcote D, Henry D. Tricyclic drugs for depression in children and adolescents. Cochrane Database Syst Rev. 2002(2):CD002317.

6. Martin A, Young C, Leckman JF, Mukonoweshuro C, Rosenheck R, Leslie D. Age effects on antidepressantinduced manic conversion. Arch Pediatr Adolesc Med. 2004;158:773-80.

7. Safer DJ, Zito JM. Treatment-emergent adverse events from selective serotonin reuptake inhibitors by age group: children versus adolescents. J Child Adolesc Psychopharmacol. 2006;16:159-69.

8. Emslie G, Kratochvil C, Vitiello B, Silva S, Mayes T, McNulty $S$, et al. Treatment for Adolescents with Depression Study (TADS): safety results. J Am Acad Child Adolesc Psychiatry. 2006;45:1440-55.

9. Gordon M, Melvin G. Selective serotonin re-uptake inhibitors-a review of the side effects in adolescents. Aust Fam Physician. 2013;42:620-3.

10. Riddle MA, King R, Hardin MT, Scahill L, Ort S, Chappell $\mathrm{P}$, et al. Behavioral side effects of fluoxetine in children and adolescents. J Child Adolesc Psychopharmacol. 1991;1:193-8.

11. Offidani E, Fava GA, Tomba E, Baldessarini RJ. Excessive mood elevation and behavioral activation with antidepressant treatment of juvenile depressive and anxiety disorders: a systematic review. Psychother Psychosom. 2013;82(3):132-41.

[Class II] This review conpares rates of excessive arousal-activation during treatment with antidepressants.

12. Joseph MF, Youngstrom EA, Soares JC. Antidepressantcoincident mania in children and adolescents treated with selective serotonin reuptake inhibitors. Future Neurol. 2009;4:87-102.
13. Wong IC, Besag FM, Santosh PJ, Murray ML. Use of selective serotonin reuptake inhibitors in children and adolescents. Drug Saf. 2004;27:991-1000.

14. Birmaher B, Axelson DA, Monk K, Kalas C, Clark DB, Ehmann M, et al. Fluoxetine for the treatment of childhood anxiety disorders. J Am Acad Child Adolesc Psychiatry. 2003;42:415-23.

15. Geller DA, Wagner KD, Emslie G, Murphy T, Carpenter DJ, Wetherhold E, et al. Paroxetine treatment in children and adolescents with obsessive-compulsive disorder: a randomized, multicenter, double-blind, placebo-controlled trial. J Am Acad Child Adolesc Psychiatry. 2004;43:1387-96.

16. Hammad TA, Laughren T, Racoosin J. Suicidality in pediatric patients treated with antidepressant drugs. Arch Gen Psychiatry. 2006;63:332-9.

17. Goodman WK, Murphy TK, Storch EA. Risk of adverse behavioral effects with pediatric use of antidepressants. Psychopharmacology (Berl). 2007;191:87-96.

18. Jick H, Kaye JA, Jick SS. Antidepressants and the risk of suicidal behaviors. JAMA. 2004;292:338-43.

19. King RA, Riddle MA, Chappell PB, Hardin MT, Anderson GM, Lombroso P, et al. Emergence of selfdestructive phenomena in children and adolescents during fluoxetine treatment. J Am Acad Child Adolesc Psychiatry. 1991;30:179-86.

20. Reinblatt SP, DosReis S, Walkup JT, Riddle MA. Activation adverse events induced by the selective serotonin reuptake inhibitor fluvoxamine in children and adolescents. J Child Adolesc Psychopharmacol. 2009;19:119-26.

21. Strawn JR, Adler CM, McNamara RK, Welge JA, Bitter SM, Mills NP. Antidepressant tolerability in anxious and depressed youth at high risk for bipolar disorder: a prospective naturalistic treatment study. Bipolar Disord. 2014;16(5):523-30.

[Class II] This article prospectively examine the rates of adverse events of antidepressants in children and adolescents who are diagnosed with depressive or anxiety disorders and have a parent with bipolar I disorder. Special focus was placed to adverse events leading to drug discontinuation.

22. Wegerer V, Moll GH, Bagli M, Rothenberger A, Rüther E, Huether G. Persistently increased density of serotonin transporters in the frontal cortex of rats treated with fluoxetine during early juvenile life. J Child Adolesc Psychopharmacol. 1999;9:13-26. 
23. Carrey NJ, Dursun S, Clements R, Renton K, Waschbusch D, MacMaster FP. Noradrenergic and serotonergic neuroendocrine responses in prepubertal, peripubertal, and postpubertal rats pretreated with desipramine and sertraline. J Am Acad Child Adolesc Psychiatry. 2002;41:999-1009.

24. Wilens TE, Biderman J, Kwon A, Chase R, Greenberg L, Mick E, et al. A systematic chart review of the nature of psychiatric adverse events in children and adolescents treated with selective serotonin reuptake inhibitors. J Child Adolesc Psychopharmacol. 2003;13:143-52.

25. Walkup J, Labellarte M. Complications of SSRI treatment. J Child Adolesc Psychopharmacol. 2001;11:1-4.

26. The Research Unit on Pediatric Psychopharmacology Anxiety Study Group. Fluvoxamine for the treatment of anxiety disorders in children and adolescents. N Engl J Med. 2001;344(17):1279-85.

27. Akagi H, Kumar TM. Lesson of the week: akathisia: overlooked at a cost. BMJ. 2002;324:1506-7.

28. Hansen L. A critical review of akathisia, and its possible association with suicidal behaviour. Hum Psychopharmacol. 2001;16:495-505.

29. Miller CH, Fleischhacker WW. Managing antipsychoticinduced acute and chronic akathisia. Drug Saf. 2000;22:73-81.

30. Bridge JA, Iyengar S, Salary CB, Barbe RP, Birmaher B, Pincus HA, et al. Clinical response and risk for reported suicidal ideation and suicide attempts in pediatric antidepressant treatment: a meta-analysis of randomized controlled trials. JAMA. 2007;297:1683-96.

31. Cooper WO, Callahan ST, Shintani A, Fuchs DC, Shelton RC, Dudley JA, et al. Antidepressants and suicide attempts in children. Pediatrics. 2014;133(2):204-10.

[Class III] This retrospective cohort study compares the risk for medically treated suicide attempts among new users of sertraline, paroxetine, citalopram, escitalopram, and venlafaxine to risk for new users of fluoxetine.

32. Libby AM, Brent DA, Morrato EH, Orton HD, Allen R, Valuck RJ. Decline in treatment of pediatric depression after FDA advisory on risk of suicidality with SSRIs. Am J Psychiatry. 2007;164:884-91.

33. Carlson GA, Mick E. Drug-induced disinhibition in psychiatrically hospitalized children. J Child Adolesc Psychopharmacol. 2003;13:153-63.

34. Vitiello B, Silva SG, Rohde P, Kratochvil CJ, Kennard BD, Reinecke MA, et al. Suicidal events in the treatment for adolescents with depression study (TADS). J Clin Psychiatry. 2009;70:741-7.

35. Guile JM. Sertraline-induced behavioral activation during the treatment of an adolescent with major depression. J Child Adolesc Psychopharmacol. 1996;6:281-5.

36. Brosen K. Some aspects of genetic polymorphism in the biotransformation of antidepressants. Therapie. 2004;59:5-12.

37. Kronenberg S, Apter A, Brent D, Schirman S, Melhem N, Pick N, et al. Serotonin transporter polymorphism (5-HTTLPR) and citalopram effectiveness and side effects in children with depression and/or anxiety disorders. J Child Adolesc Psychopharmacol. 2007;17:741-50.

38. Gerber PE, Lynd LD. Selective serotonin-reuptake inhibitor-induced movement disorders. Ann Pharmacother. 1998;32:692-8.

39. Lim CJ, Leckman JF, Young C, Martin A. Antidepressant-induced manic conversion: a developmentally informed synthesis of the literature. Int Rev Neurobiol. 2005;65:25-52.

40. Cohen D. Should the use of selective serotonin reuptake inhibitors in child and adolescent depression be banned? Psychother Psychosom. 2007;76:5-14.

41. Walkup J, Labellarte M, Riddle MA, Pine DS, Greenhill L, Fairbanks J, et al. Treatment of pediatric anxiety disorders: an open-label extension of the research units on pediatric psychopharmacology anxiety study. J Child Adolesc Psychopharmacol. 2002;12:175-88.

42. Birmaher B, Axelson D. Course and outcome of bipolar spectrum disorder in children and adolescents: a review of the existing literature. Dev Psychopathol.

2006;18:1023-35.

43. Gilman SE, Dupuy JM, Perlis RH. Risks for the transition from major depressive disorder to bipolar disorder in the national epidemiologic survey on alcohol and related conditions. J Clin Psychiatry. 2012;73:829-36.

44. Blier P, de Montigny C. Possible serotonergic mechanisms underlying the antidepressant and antiobsessive-compulsive disorder responses. Biol Psychiatry. 1998;44:313-23.

45. Lopez JP, Lim R, Cruceanu C, Crapper L, Fasano C, Labonte B. miR-1202 is a primate-specific and brain-enriched microRNA involved in major depression and antidepressant treatment. Nat Med. 2014;20:764-8. 